\title{
Self-organized plasmonic metasurfaces for all-optical modulation
}

\author{
G. Della Valle,${ }^{1,2,{ }^{*}}$ D. Polli, ${ }^{1,2}$ P. Biagioni, ${ }^{1}$ C. Martella, ${ }^{3}$ M. C. Giordano,${ }^{3}$ M. Finazzi, ${ }^{1}$ \\ S. Longhi, ${ }^{1,2}$ L. Duò, ${ }^{1}$ G. Cerullo, ${ }^{1,2}$ and F. Buatier de Mongeot $^{3}$ \\ ${ }^{1}$ Dipartimento di Fisica, Politecnico di Milano, Piazza L. da Vinci 32, I-20133 Milano, Italy \\ ${ }_{2}^{2}$ Istituto di Fotonica e Nanotecnologie, Consiglio Nazionale delle Ricerche, Piazza L. da Vinci 32, I-20133 Milano, Italy \\ ${ }^{3}$ Dipartimento di Fisica, Università di Genova, and CNISM, Via Dodecaneso 33, I-16146 Genova, Italy
}

(Received 6 February 2015; revised manuscript received 22 May 2015; published 23 June 2015)

\begin{abstract}
We experimentally demonstrate a self-organized metasurface with a polarization dependent transmittance that can be dynamically controlled by optical means. The configuration consists of tightly packed plasmonic nanowires with a large dispersion of width and height produced by the defocused ion-beam sputtering of a thin gold film supported on a silica glass. Our results are quantitatively interpreted according to a theoretical model based on the thermomodulational nonlinearity of gold and a finite-element numerical analysis of the absorption and scattering cross-sections of the nanowires. We found that the polarization sensitivity of the metasurface can be strongly enhanced by pumping with ultrashort laser pulses, leading to potential applications in ultrafast all-optical modulation and switching of light.
\end{abstract}

DOI: 10.1103/PhysRevB.91.235440

PACS number(s): 73.20.Mf, 78.47.J-, 79.20.Rf, 81.16.Rf

\section{INTRODUCTION}

During the last decade, noble metal nanostructures have received an increasing attention because of their unique capability to spatially localize optical near-fields well beyond the diffraction limit, thanks to the excitation of plasmonpolariton resonances [1]. This fundamental phenomenon can be exploited to manipulate light-matter interaction in ways that are impossible to achieve with purely dielectric materials, and a plethora of plasmonic structures have been so far reported within a broad landscape of applications, from sensing to waveguiding (see, e.g., Refs. [2-4] and references therein). In particular, ultrathin layers of plasmonic nanostructures, closely packed to form a metasurface [5], can operate as surfaceconfined flat optical components for polarization management [6-8], negative reflection and refraction [9] and analog computing [10], just to mention a few linear functionalities that have been recently demonstrated. Most interestingly, plasmonic metasurfaces have also been proposed to enhance the optical nonlinearity of noble metals, thus disclosing the novel research branch of nonlinear plasmonics [11]. In fact, it is very well known that gold and silver can exhibit a huge thermomodulational optical nonlinearity [12,13], but the high Ohmic losses have prevented bulk noble metals from becoming nonlinear optical media. On the contrary, plasmonic resonances in metasurfaces can be exploited to increase the optical transmission as compared with thin metallic films and simultaneously enhance the nonlinear interaction thanks to the high local fields. This is especially important for applications in the red and near infrared, where the intrinsic nonlinearity of noble metals is much weaker as compared with the peak values achievable close to interband transitions, falling in the green spectral region for gold [14]. The search for the optimal configuration of metasurfaces capable of providing sufficient nonlinear enhancement in the desired spectral band, integrability with more conventional optical technologies and, last but not least, ease of fabrication and high stability of large

*Corresponding author: giuseppe.dellavalle@polimi.it area samples, is still an open challenge. Very promising results have been reported for metasurfaces made of patterned gold nanowires produced by a top-down lithographic technique [15] or vertical gold nanopillars synthesized by bottom-up electrochemical means [16]. Self-assembly and self-organization of plasmonic nanostructures have also attracted an increasing interest in the last years, with the demonstration of high-quality clusters [17] and arrays [18] of nanoparticles and potential application to metamaterials [19]. However, to the best of our knowledge, no evidence of a substantial enhancement of the thermomodulational nonlinearity of noble metals has been so far reported for self-assembled or self-organized plasmonic metasurfaces.

Here, we study the linear and nonlinear optical properties of self-organized plasmonic metasurfaces produced by defocused ion beam sputtering of a thin gold film supported on a silica substrate. We demonstrate that these structures can be described as an ensemble of parallel metallic nanowires with broad size distribution. For excitation with polarization perpendicular to the major axis of the wires, the metasurface displays a strong plasmonic resonance in the near-infrared. We show, by a combination of experiments and modeling, that upon ultrashort pulse excitation these structures exhibit a macroscopic variation in gold permittivity, resulting in a sizable and ultrafast transmission change. Due to the ease of fabrication of large-area structures and to the peculiar nonlinear optical modulation properties, we believe that these self-organized plasmonic metasurfaces represent a promising new route towards the realization of low-cost ultracompact high-speed plasmon-based optical modulators and switches.

\section{SAMPLE FABRICATION AND CONTINUOUS-WAVE OPTICAL RESPONSE}

Gold nanowires (NWs) are grown on silica by means of a self-organized approach based on defocused ion beam sputtering (IBS) [20,21]. The experimental procedure is schematically sketched in Fig. 1(a): after thermal deposition of a 150 -nm-thick polycrystalline gold film on a silica substrate kept at room temperature, IBS is performed at grazing 
(a)
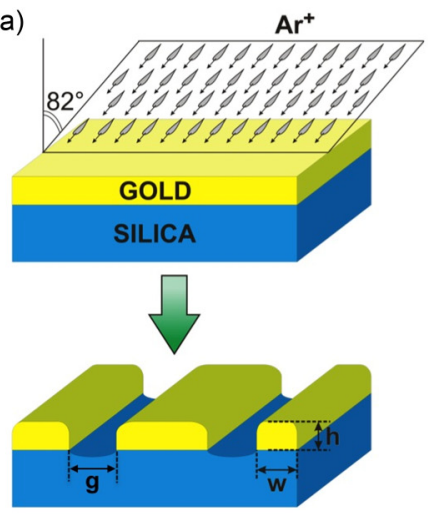

(b)

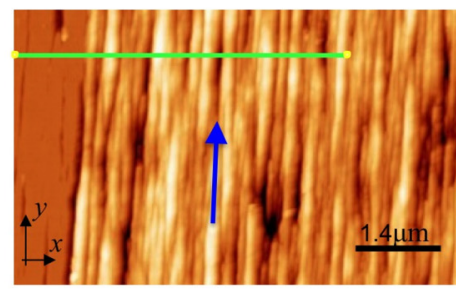

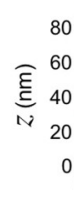

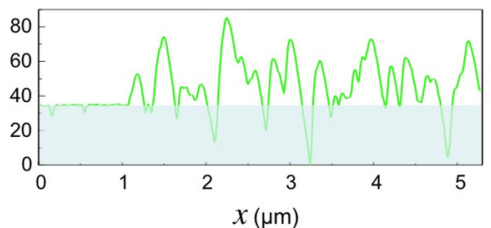

$z(\mathrm{~nm})$

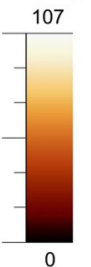

0

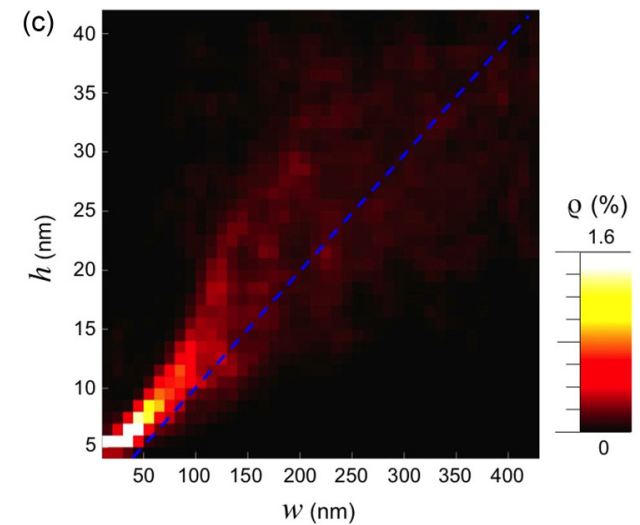

FIG. 1. (Color online) (a) Sketch of the $\mathrm{Ar}^{+}$IBS technique employed to produce self-organized gold NWs on a silica substrate. (b) The AFM map of the sample acquired across a mechanical scratch made on the gold metasurface evidences the underlying silica substrate exposed on the left side. The blue arrow indicates the ion beam projection direction. In the inset, a typical height profile acquired along the cross section shown in the AFM map. Shaded light blue area represents the region of the glass substrate. (c) Size distribution $\rho(w, h)$ of NW width $w$ and height $h$ for the sample considered in (b). Dashed blue line is the locus of $w / h=10$ aspect ratio.

incidence $\left(82^{\circ}\right)$ with a high flux defocused $\mathrm{Ar}^{+}$beam at $800-\mathrm{eV}$ energy (ion flux $\sim 4.95 \times 10^{15}$ ions $\mathrm{s}^{-1} \mathrm{~cm}^{-2}$ ) in a custom made ultrahigh vacuum chamber. Owing to the grazing ion incidence conditions, for low fluencies in the range of $10^{16}-10^{17}$ ions $\mathrm{cm}^{-2}$, a self-organized ripple pattern develops at the metal/vacuum interface with the ridges aligned along the beam projection [blue arrow in Fig. 1(b)] as a result of the competition between the erosive action of the ions and the smoothing mechanism triggered by atom diffusion. When ion bombardment is prolonged up to fluencies in the range of $10^{18}$ ions $\mathrm{cm}^{-2}$, the bottoms of the valleys reach the dielectric substrate, and the ripple pattern decomposes into an array of laterally disconnected metal NWs, as highlighted in the atomic force microscopy (AFM) topography of Fig. 1(b). From this erosion stage onwards, the excitation of localized surface plasmon resonances becomes possible when the light is polarized perpendicularly to the NWs [22]. In particular, in Fig. 1(b), one can easily observe the degree of disconnection of the metal nanostructures thanks to the AFM image acquired across a mechanical scratch of the gold pattern. Since the underlying silica substrate is exposed, it is possible to simultaneously visualize it with the gold NWs [21]. In order to measure for each AFM image the size distribution $\rho(w, h)$ of NWs width $w$ and height $h$, we developed a MATLAB code, which adopts the base level of the silica substrate as a reference threshold for measuring the height of individual NWs. As shown in Fig. 1(c), while the $(w, h)$ distribution of the NWs measured in the transverse direction is large, their aspect ratio $w / h$ remains well defined around 10 throughout the whole range, whereas the average width turns out to be $w_{\text {ave }} \simeq 175 \mathrm{~nm}$. We also investigated the statistics of the gap $g$, which is the distance between the edges of adjacent nanowires, and found an average value $g_{\text {ave }} \simeq 50 \mathrm{~nm}$. Along the longitudinal direction the NWs instead form a connected network, which allows macroscopic charge transport with remarkably low sheet resistance up to the highest ion fluencies [23]. Typical values of the longitudinal sheet resistance are found in the range $1-5 \Omega / \mathrm{sq}$, while the transverse sheet resistance can be two orders of magnitude larger. Nanofabrication via IBS thus allows one to create a plasmonic metasurface made of disconnected gold NWs with high aspect ratio in the wire cross section and large dispersion of widths and heights.

The continuous-wave optical response of the metasurface was investigated by recording the transmission spectrum of the sample under illumination at normal incidence with linearly polarized light. Results are reported in Fig. 2(a) for two orthogonal polarizations, i.e., with incident electric field $E_{0}$ parallel (solid black line) or orthogonal (solid red line) to the major axis of the NWs. The difference between the two spectra reveals a polarization sensitivity of the metasurface connected with the orientation of the NWs, with the formation of a broad dip in the $E_{0} \perp$ transmission centered at around $820 \mathrm{~nm}$.

To understand the origin of such polarization dependent transmission we developed a model of the linear optical response of the metasurface. In order to make the problem easier to handle, we introduced the following simplifying hypotheses: (i) the NWs are considered to be infinitely long in the $y$ direction [cf. inset in Fig. 2(a)], allowing one to avoid 3D modeling in favour of a 2D modeling; (ii) near-field interaction among neighboring NWs is disregarded; and (iii) superposition of the fields scattered by the NWs in the far field is assumed to be incoherent. The first hypothesis is based on the fact that the major axis of the NWs is of the order of few micrometers, i.e., of the order of the propagation distance of the plasmonic waves sustained by the NWs [24,25]. It is therefore expected that the onset of Fabry-Perot (i.e., retardation-based) plasmonic resonances along the major axis would be located in the midinfrared, having a negligible impact onto the optical response in the visible and near infrared. The second hypothesis is based on the observation that neighboring NWs are typically very different in width and height, so that their plasmonic resonances are strongly detuned from each other and thus very weakly coupled. The third hypothesis is due to the fact that the overall structure lacks any long-range ordering, so interference effects in the far-field are expected to be negligible, similar to what observed in an ensemble of plasmonic nanoparticles dispersed in a liquid. According to the above simplifications, the optical response of the metasurface was modeled as an incoherent superposition of the individual optical responses of several different infinitely long plasmonic 

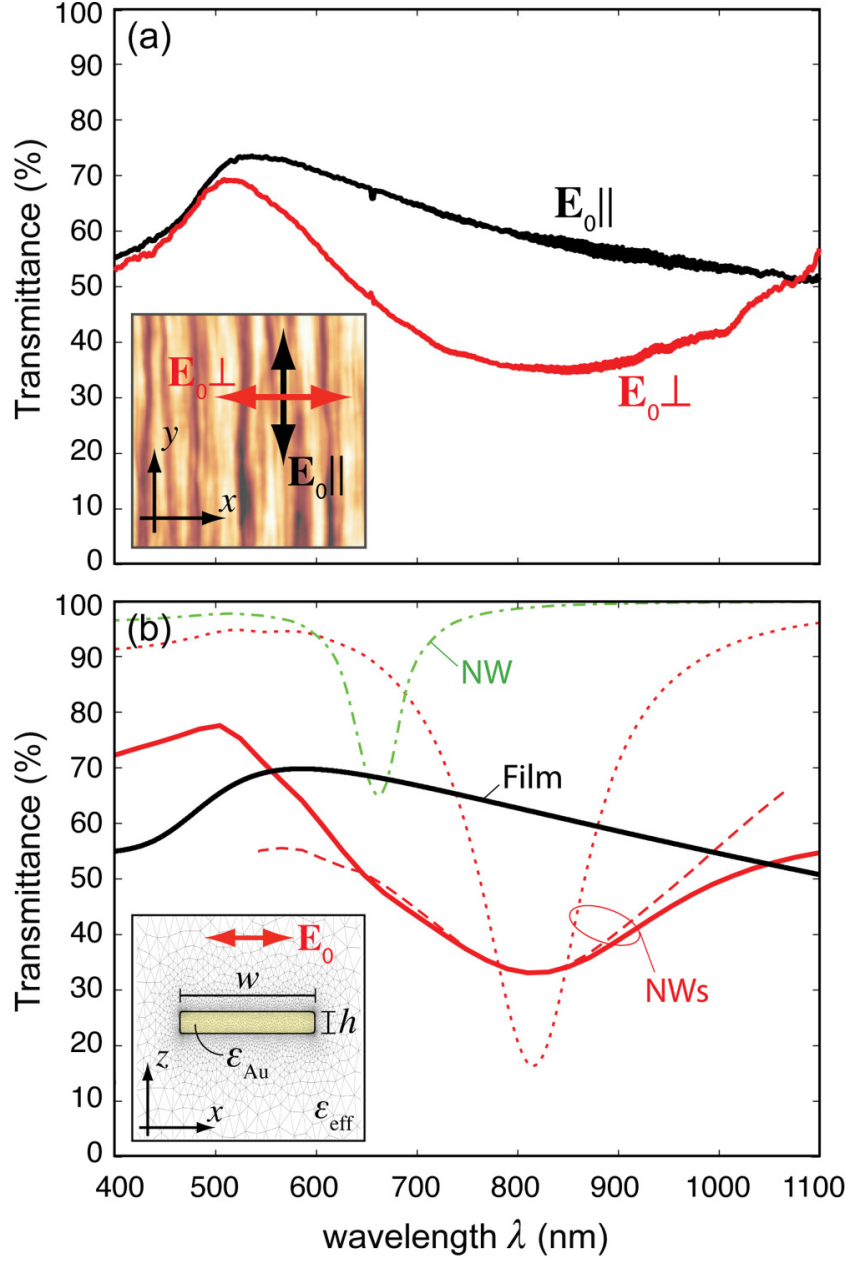

FIG. 2. (Color online) (a) Polarization dependent experimental transmittance of the self-organized plasmonic metasurface. The inset shows the directions of the incident electric field corresponding to the two orthogonal polarizations. (b) Numerically computed transmission spectrum of (solid black) a uniform 5-nm-thin gold film on silica and (solid red) an ensemble of gold NWs with $w-h$ dispersion as in Fig. 1(c). Panel (b) also shows the transmittance of an ensemble of identical NWs with: (dash-dotted green) $w=50 \mathrm{~nm}$ and $h=6 \mathrm{~nm}$; (dotted red) $w=110 \mathrm{~nm}$ and $h=8.5 \mathrm{~nm}$; (dashed red) $w=110 \mathrm{~nm}$, $h=8.5 \mathrm{~nm}$, and effective artificial broadening mimicking the optical response of the dispersed ensemble. The inset details the 2D geometry employed for FEM simulations of the absorption and scattering cross-sections of each $w$ - $h$ population of NWs.

NWs aligned to each other and distributed in width and height according to the statistics detailed in Fig. 1(c). More precisely, we first computed, by finite-element method (FEM) numerical simulations [26] [with the 2D geometry shown in the inset of Fig. 2(b)], the total extinction cross-section spectrum $\sigma_{E}(\lambda ; w, h)$ of each gold NW [27] with a given width $w$ and height $h$. In total, we computed 1677 different configurations, corresponding to the number of pixels in the map of Fig. 1(c). For simplicity, in our simulations we assumed the NW to be embedded in a homogeneous medium with an effective permittivity taken as the average value between the ones of the fused-silica substrate and air, i.e., $\epsilon_{\text {eff }}=1.56$. Then, considering a collimated incident Gaussian beam with diameter $D_{0}$ and power $P_{0}$, the total power absorbed or scattered by the NW ensemble is computed as

$$
P_{E}(\lambda)=\xi N I_{0} \sum_{w, h} \rho(w, h) \sigma_{E}(\lambda ; w, h),
$$

where $I_{0}=P_{0} / D_{0}$ is the average incident intensity and $N=D_{0} / w_{d}$ is the average number of NWs exposed to the incident beam, with $w_{d}=w_{\text {ave }}+g_{\text {ave }} \simeq 225 \mathrm{~nm}$ an estimation of the average distance between two adjacent NWs. The adimensional parameter $\xi$ in Eq. (1) is a fitting constant of the order of 1 , taking into account quantitative deviations of the model due to the simplifications above detailed. Note that, since our model is in $2 \mathrm{D}$, the NW cross sections are lengths and not areas, and the intensity $I_{0}$ is measured in $\mathrm{W} / \mathrm{m}$. Also, despite of the fact that the size distribution $\rho$ of Fig. 1(c) indicates major abundance of small NWs, we found that the whole set of sizes contributes to the total optical response and ought to be included in the calculations (see Appendix A for details). According to Eq. (1), the theoretical transmission spectrum of the metasurface is computed as

$$
T(\lambda)=1-\frac{P_{E}(\lambda)}{P_{0}}=1-\frac{\xi}{w_{\mathrm{d}}} \sum_{w, h} \rho(w, h) \sigma_{E}(\lambda ; w, h) .
$$

Results of Eq. (2) for the case of polarization orthogonal to the NWs and with $\xi=0.31$ are shown in Fig. 2(b) (solid red curve). Note the good agreement with the experimental spectrum of Fig. 2(a) (solid red curve). In particular, the model is capable of capturing the formation of the broad transmission dip at around $820 \mathrm{~nm}$. Interestingly, this dip cannot be explained in terms of the optical response of the most representative NW in the distribution in Fig. 1(c), having $w=50 \mathrm{~nm}$ and $h=6 \mathrm{~nm}$, since its spectral response is peaked at around $670 \mathrm{~nm}$ [dash-dotted green curve in Fig. 2(b)]. On the contrary, the dip observed at $820 \mathrm{~nm}$ would correspond to the plasmonic resonance of a single-sized ensemble of identical noninteracting NWs with $w=w_{0}=110 \mathrm{~nm}$ and $h=h_{0}=8.5 \mathrm{~nm}$, although the width and depth of the dip would be very inaccurate [dotted red curve in Fig. 2(b)]. However, a good quantitative agreement can be retrieved provided that an extra broadening of the plasmonic resonance is artificially introduced, increasing by $\Delta \epsilon_{I}$ the imaginary part of the gold permittivity. This way, one can compute by FEM a broadened extinction cross-section $\hat{\sigma}_{E}(\lambda)$ of the $\left(w_{0}, h_{0}\right) \mathrm{NW}$, and thus the transmittance spectrum of the metasurface as

$$
T(\lambda)=1-\frac{\xi^{\prime}}{w_{0}+g_{\text {ave }}} \hat{\sigma}_{E}(\lambda) .
$$

Results from Eq. (3) in the wavelength range 550-1050 nm with $\Delta \epsilon_{I}=15$ and $\xi^{\prime}=1.8$ are shown as a dashed curve in Fig. 2(b). Note that a different value of the fitting parameter is needed here to retrieve a good quantitative agreement with the experimental data. This is due to the fact that, contrary to the model of Eq. (2), in Eq. (3) the contribution from very wide NWs is totally disregarded (see Appendix A for further details). Of course, Eq. (3) is a heuristic approximation of the actual transmittance, which is unable to reproduce the whole spectrum but only the main plasmonic feature observed at around $820 \mathrm{~nm}$. However, this model retains the basic 
electromagnetic properties of the original model of Eq. (2), but it is much more tractable [28] for tackling the problem of simulating the transient optical response of the metasurface, and will be exploited in Sec. III B. As a final remark, it is worth saying that a different simplified model providing similar results can be developed considering a homogeneous array of interacting identical NWs. In this model, the broadening of the plasmonic response is retrieved by introducing the near-field coupling between the wires. However, to quantitatively reproduce the correct transmittance, some heuristic corrections ought to be introduced similarly to the case of model Eq. (3) (see Appendix B for further details).

For parallel polarization, again one can compute the extinction cross-section of individual NWs and then apply superposition effects as detailed by Eqs. (1) and (2). However, in this case the model can be further simplified, in view of the fact that the NWs are considered to be infinitely long in the $y$ direction [our hypothesis (i)], and the metasurface can be effectively reduced to a thin gold film of suitable effective thickness. This way, the theoretical transmission can be computed by the transfer matrix method (see, e.g., Ref. [29]). Results are shown in Fig. 2(b) (solid black curve) for an effective thickness equal to $5 \mathrm{~nm}$. To mimic the scattering losses caused by the inhomogeneities of the metasurface we heuristically added $20 \%$ extra losses to the ideal 5-nm-thick homogenous film. This way the theoretical transmission turns out to be in good quantitative agreement with the experimental transmission for parallel polarization [solid black curve in Fig. 2(a)].

According to our modeling, we conclude that the CW optical response of the self-organized metasurface is dominated by an ultra broadband plasmonic resonance peaked at $820 \mathrm{~nm}$ for incident electric field orthogonal to the NW major axis. This resonance is the result of incoherent superposition of extinction spectra from a broadly dispersed population of NWs with different widths and heights and a relatively uniform aspect ratio $w / h$. On the contrary, no plasmonic feature is present under excitation with incident electric field parallel to the NW major axis. In the latter case, the metasurface behaves like a homogenous gold film of suitable thickness.

Finally, it is worth noticing that in all the simulations, the gold permittivity $\epsilon_{\mathrm{Au}}(\lambda)$ was taken as a cubic interpolation of the experimental data from Johnson and Christy [30]. Corrections to the Drude damping due to the extra scattering mechanisms taking place in nanostructures (see, e.g., Refs. [31-33]) and due to surface roughness scattering have been disregarded in the modeling of the NWs. However, such approximations only have minor effects on the final result, since the broadening in the plasmonic spectrum of the metasurface is dominated by inhomogeneous mechanisms, due to the size dispersion of the NWs.

\section{TRANSIENT OPTICAL RESPONSE}

\section{A. Experiments}

To investigate the transient optical response of the metasurface and its capability to provide all-optical modulation and switching, we employed ultrafast pump-probe spectroscopy [34]. Our setup is based on an amplified Ti:sapphire laser
(Libra-HE from Coherent) producing 100-fs, 4-mJ pulses at $800 \mathrm{~nm}$ and $1-\mathrm{kHz}$ repetition rate. The metasurface sample, mounted onto a rototranslational stage, is excited by $100-\mathrm{fs}$ pulses at the laser fundamental wavelength, and probed by white-light continuum pulses generated in a sapphire plate, with spectrum covering the 400-1100 nm wavelength range. The white-light continuum is not compressed, resulting in a $~ 400$-fs chirp, which has been corrected by data postprocessing. Pump and probe beams are linearly polarized along the same direction. The desired polarization conditions on the sample are obtained by acting on the rotation axis of the stage to impose incidence with parallel or orthogonal polarizations with respect to the major axis of the NWs. Broadband detection is achieved with an optical multichannel analyzer (Stresing Entwicklungsbüro) working at the full $1-\mathrm{kHz}$ laser repetition rate [35]. The measured signal is a two-dimensional map of the differential transmission $\Delta T / T$ as a function of the probe wavelength $\lambda$ and the pump-probe time delay $t: \Delta T / T(\lambda, t)=T_{\text {on }}(\lambda, t) / T_{\text {off }}(\lambda)-1$, where $T_{\text {on }}$ and $T_{\text {off }}$ are the probe spectra transmitted through the excited and the unexcited sample, respectively. Due to the need to filter out the intense residual 800-nm component in the white light, the 780-820-nm spectral range is not available in the $\Delta T / T$ spectra. The instrumental response function of the setup has a full width at half maximum of $130 \mathrm{fs}$.

Experimental results for incident pump fluence $F=$ $0.6 \mathrm{~mJ} / \mathrm{cm}^{2}$ are reported in Figs. 3(a) and 3(b) for polarizations parallel and orthogonal to the NWs, respectively. The probe fluence is $<1 \mu \mathrm{J} / \mathrm{cm}^{2}$ per $10 \mathrm{~nm}$ of bandwidth.

The map measured for parallel polarization [Fig. 3(a)] is dominated by two lobes in the 430-550-nm spectral region, one positive, peaked at around $460 \mathrm{~nm}$, and one negative, peaked at around $510 \mathrm{~nm}$, with negligible signal in the red and infrared part of the spectrum. These two lobes exhibit similar dynamics, reaching their maxima (in absolute value) at around 0.5-ps pump-probe delay and then decaying to a long-living plateau within about 5-6 ps. Very similar features are observed in the 430-550-nm range also for the orthogonal polarization [Fig. 3(b)], but with the addition of an extra feature dominating the red and infrared part of the spectrum, consisting of a broad positive lobe centered at around $700 \mathrm{~nm}$ and a much weaker and even broader negative lobe centered at around $1000 \mathrm{~nm}$. Again these two lobes follow a similar dynamics, reaching their maxima (in absolute value) at around 0.5 -ps time delay and then decaying to a long-living plateau within about 5-6 ps. More quantitatively, it is interesting to note that for the orthogonal polarization [Fig. 3(b)] the $\Delta T / T$ peak at $700 \mathrm{~nm}$ is about $2 \%$, whereas at this same wavelength (and under the same incident pump fluence) the $\Delta T / T$ for the parallel polarization [Fig. 3(a)] is about $0.1 \%$, i.e., 20 times smaller. Such a discrepancy between the two polarizations turns out to be much higher as compared with the one observed in the continuous wave regime: at $750 \mathrm{~nm}$, where the difference is more pronounced in the linear spectra of Fig. 2(a), the transmittance changes from $62 \%$ for the parallel polarization to $38 \%$ for the orthogonal polarization. Therefore it is found that the polarization sensitivity of the metasurface is strongly enhanced in the nonlinear regime after excitation with fs-pump pulses as compared with the linear continuous-wave regime. 

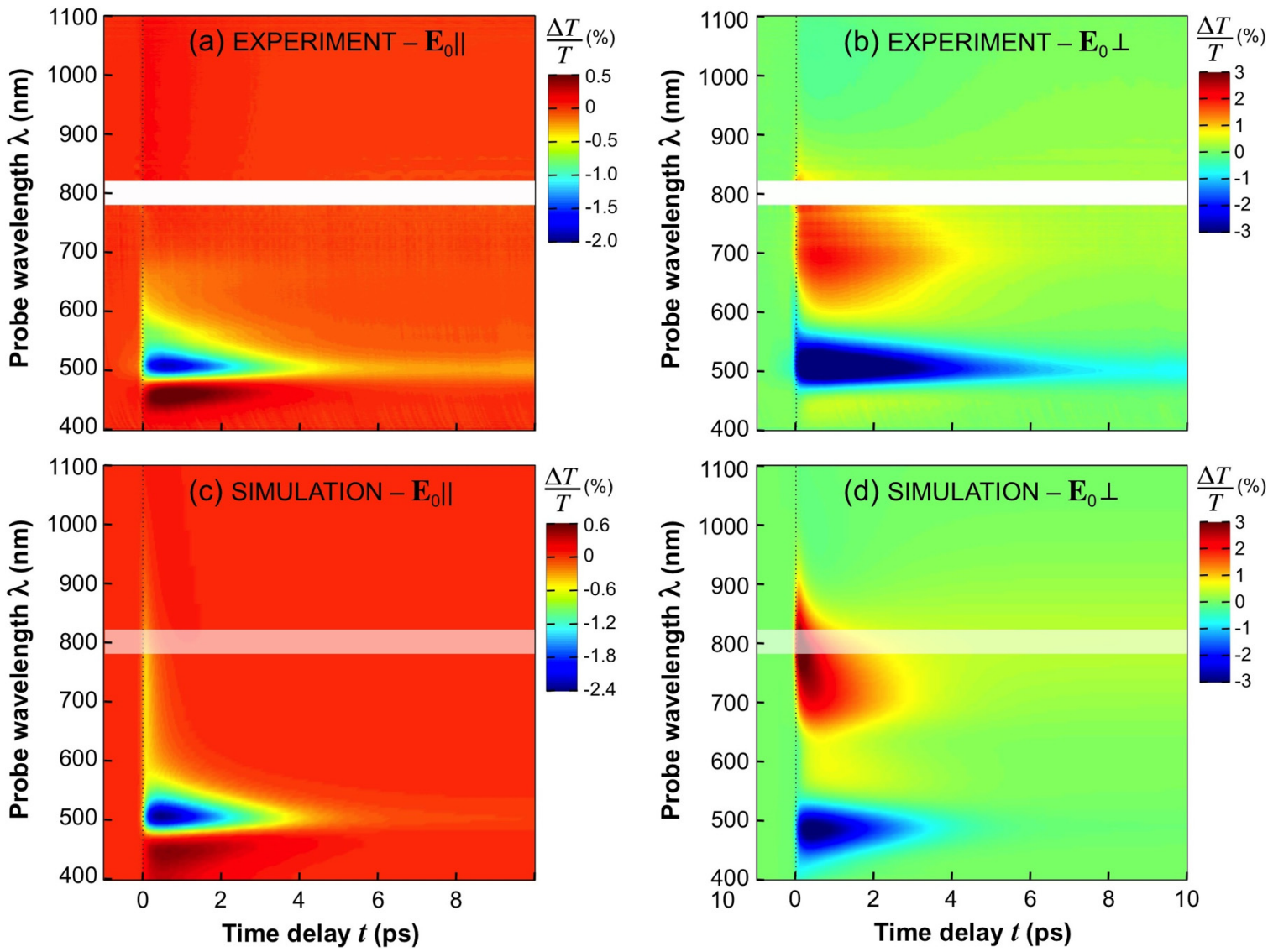

FIG. 3. (Color online) Experimental differential transmission map of the metasurface for (a) parallel polarization ( $\left.\mathbf{E}_{0}||\right)$ and (b) orthogonal polarization $\left(\mathbf{E}_{0} \perp\right)$. Theoretical differential transmission map of (c) a uniform 5-nm-thin gold film on silica for parallel polarization and (d) the effectively broadened gold NW with $w=110 \mathrm{~nm}$ and $h=8.5 \mathrm{~nm}$ for orthogonal polarization. Incident pump fluence $F=0.6 \mathrm{~mJ} / \mathrm{cm}^{2}$ for both experiments and simulations.

\section{B. Simulations}

To understand the origin of the $\Delta T / T$ enhancement for polarization orthogonal to the NWs, and, more generally, to provide a physical explanation of the several features observed in Figs. 3(a) and 3(b) (especially the appearance of the two lobes in the red and infrared spectrum under orthogonal polarization, having no counterpart for parallel polarization), we developed a model of the pump-probe experiments. We followed the approach reported in previous papers on thin gold films [36] and multilayers [37] (see also original works by Sun, Vallée and colleagues [14,38]). The key feature of this model is a semiclassical description of the microscopic dynamical processes taking place in noble metals after irradiation with fs pump pulses. Provided that the pump photon energy lies below the interband absorption edge of the metal (as in the present case), pump absorption takes place by means of plasmonic excitation, involving free electrons in the conduction band. After few fs, the plasmon dephasing leaves the electronic population in a highly nonthermal state, i.e., a population of nonthermalized electrons is created. This population then releases its excess energy (by electron-electron scattering) to the thermalized electronic population which is thus heated up from room temperature $T_{0}$ to a higher temperature $T_{E}$. Then, electron-phonon interaction, taking place on the picosecond time scale, causes the lattice temperature $T_{L}$ to increase at the expense of the electronic temperature. Finally, the sample cools down to the room temperature by phonon-phonon interactions with the environment, a process taking place on a much longer timescale (of the order of $100 \mathrm{ps).} \mathrm{The} \mathrm{time-}$ dependent variations of (either thermalized or nonthermalized) electronic populations give rise to a modulation of interband optical transitions (so-called Fermi smearing mechanism), and thus of interband permittivity of gold. Also, the lattice temperature increase affects the Drude damping and the plasma frequency, causing a modulation of the free-carrier permittivity contribution. The overall variation of the gold permittivity $\Delta \epsilon_{\mathrm{Au}}(\lambda, t)$ then dynamically modifies the optical response of the system to a weak probe signal, which can be computed by solving for Maxwell's equations, depending on the geometrical structure under consideration (either a thin film, a multilayer, a plasmonic nanostructure, or a metasurface).

More quantitatively, the dynamical exchange of energy between the different degrees of freedom is detailed by the so-called two-temperature model (TTM) [39] and subsequent refinements considering a third temperature to account for nonthermalized carriers (see e.g. Refs. [14,40]), or, more accurately, many more temperatures (in fact a spectrum of energies for nonthermalized carriers) [41], depending on the desired accuracy one is aiming at. Here we adopted an 
extended TTM (ETTM), reading as follows [43]:

$$
\begin{aligned}
& C_{E} \frac{d T_{E}}{d t}=-g\left(T_{E}-T_{L}\right)+\int_{-\infty}^{+\infty} P_{a}\left(t^{\prime}\right) H\left(t-t^{\prime}\right) d t^{\prime}, \\
& C_{L} \frac{d T_{L}}{d t}=g\left(T_{E}-T_{L}\right),
\end{aligned}
$$

where $C_{E}=\gamma T_{E}$ and $C_{L}$ are the electronic and lattice heat capacities ( $\gamma$ being the so-called electron heat capacity constant), $g$ is the electron-phonon coupling constant, and $H(t)$ is a response function accounting for the energy transfer from nonthermalized electrons (generated by pump excitation) to thermalized ones, reading

$$
\begin{aligned}
H(t)= & -\frac{1}{t} e^{-\left(\frac{h v_{p}}{E_{F}}\right)^{2} \frac{t}{\tau_{0}}} \\
& \times\left\{1+\left(\frac{E_{F}}{h v_{p}}\right)^{2} \frac{\tau_{0}}{t}\left[1-e^{\left(\frac{h v_{p}}{E_{F}}\right)^{2} \frac{t}{\tau_{0}}}\right]\right\} h(t) .
\end{aligned}
$$

In Eq. (5), $h(t)$ is the step (Heaviside) function, and $h v_{p}$ is the pump photon energy (equals to $\sim 1.56 \mathrm{eV}$ in our case where $\left.\lambda_{P} \simeq 800 \mathrm{~nm}\right), E_{F}$ the Fermi energy (7.3 eV in gold) and $\tau_{0}=6$ fs (in agreement with Refs. [36,42]). The values of the other ETTM coefficients for gold can be found in Refs. $[37,43]$. Note that the ETTM is capable of capturing the effect of nonthermalized carriers without using the Boltzmann formalism [41] and thus extends the classic TTM beyond the three-temperature approach proposed in Ref. [14]. In Eq. (4), $P_{a}(t)$ is the pump pulse power absorbed in the unit volume of the metasurface, estimated according to the formula [44]:

$$
P_{a}(t)=\sqrt{\frac{4 \ln (2)}{\pi}} A\left(\lambda_{P}\right)\left(\frac{F}{h_{m} \tau_{P}}\right) \exp \left(-\frac{4 \ln (2) t^{2}}{\tau_{P}^{2}}\right),
$$

where $F$ is the incident fluence of the pump pulse, $\tau_{P}=130$ fs is the FWHM of the instrumental response function as estimated in our experiments, $h_{m}$ is the (effective) thickness of the metal, and $A\left(\lambda_{P}\right)$ is the linear absorption of the metasurface at the pump wavelength determined from numerical simulations. For parallel polarization, since the linear optical response was modeled as that of a homogenous thin gold film of 5-nm effective thickness on a silica substrate (cf. previous section), the absorption spectrum $A(\lambda)$ is computed accordingly as $A(\lambda)=1-R(\lambda)-T(\lambda)$, being $R(\lambda)$ and $T(\lambda)$ the reflection and transmission spectra of the thin film retrieved by the transfer matrix method. For orthogonal polarization, in agreement with the modeling of the linear optical response as an incoherent superposition of several different gold NWs (cf. previous section), $A(\lambda)$ is computed as

$$
A(\lambda)=\frac{\xi}{w_{d}} \sum_{w, h} \rho(w, h) \sigma_{A}(\lambda ; w, h) .
$$

Equation system (4) is solved by numerical integration using the standard Runge-Kutta method. Once the two dynamical variables $T_{E}(t)$ and $T_{L}(t)$ are retrieved from Eq. (4), the corresponding variations of the real and imaginary parts of the gold permittivity, given by $\Delta \epsilon_{\mathrm{Au}}^{\prime}(\lambda, t)$ and $\Delta \epsilon_{\mathrm{Au}}^{\prime \prime}(\lambda, t)$, respectively, are determined by following the same calculations [45] as those reported in a previous paper on gold-based Bragg filters
[37] (see also Refs. [46,47] for generalities, and Ref. [48] for details on gold parameters employed in the calculations).

Once the complex $\Delta \epsilon_{\mathrm{Au}}(\lambda, t)$ is determined, the $\Delta T / T$ maps for parallel and orthogonal polarizations are computed perturbatively (in agreement with the fact that the experimental $\Delta T / T$ of Fig. 3 is of the order of a few percent, under the considered pump fluence):

$$
\frac{\Delta T}{T}(\lambda, t)=\frac{1}{T(\lambda)}\left[\frac{d T}{d \epsilon^{\prime}}(\lambda) \Delta \epsilon_{\mathrm{Au}}^{\prime}(\lambda, t)+\frac{d T}{d \epsilon^{\prime \prime}}(\lambda) \Delta \epsilon_{\mathrm{Au}}^{\prime \prime}(\lambda, t)\right] .
$$

In Eq. (8), $T(\lambda)$ is the linear transmission spectrum of the structure given by the simplified model of Eq. (3) for orthogonal polarization, whereas for the parallel polarization $T(\lambda)$ is computed by the transfer matrix method. The spectral coefficients $d T / d \epsilon^{\prime}$ and $d T / d \epsilon^{\prime \prime}$ in Eq. (8) are numerically estimated as $\Delta T / \Delta \epsilon^{\prime}$ and $\Delta T / \Delta \epsilon^{\prime \prime}$ respectively, being $\Delta \epsilon^{\prime}=$ $\Delta \epsilon^{\prime \prime}=0.2$ a small variation of the real and imaginary part of the Au permittivity, of the order of the average variation of the permittivity of Au induced by pump absorption in the metasurface (estimated from the ETTM and the theoretical absorption of the structure under the considered pump fluence and polarization conditions). Results of the numerical simulations are reported in Figs. 3(c) and 3(d). For parallel polarization, a good quantitative agreement between the experimental pump-probe map [Fig. 3(a)] and the theoretical one [Fig. 3(c)] is retrieved on the whole spectrum of the probe. Therefore the accuracy of our modeling of the differential transmission maps further confirms that, under parallel polarization, the approximation of the metasurface as an effective thin gold film of suitable thickness is appropriate. As it is well known, pump absorption and subsequent modulation of gold interband transitions causes the appearance of a negative lobe in the $\Delta T / T$ map of thin gold films, peaked at around $500 \mathrm{~nm}$ and about 50-70 nm wide (see, e.g., Refs. [14,36]). At shorter wavelengths a weaker positive lobe appears, with comparable width, whereas at longer wavelengths the spectrum is almost flat and decreases to zero very smoothly [14,37]. The two lobes in the 400-600-nm wavelength range directly follow from the smearing of the electrons energy distribution around the Fermi level and the excitation of nonthermalized carriers, induced by the heating of conduction electrons after pump absorption (see Refs. [14,36,37] for details). Since these same spectral features are present in the pump-probe map of the metasurface under parallel polarization [Fig. 3(a)], they can be attributed to the same mechanisms taking place in a thin gold film, and thus have no relation with the plasmonic resonances of the metasurface. More interesting is the case of the orthogonal polarization: the appearance of the two lobes in the red-infrared spectral region is confirmed in our simulations [Fig. 3(d)], thus indicating that the approach of modeling the metasurface as an effective ensemble of identical NWs with suitable width and height and an extra broadening (to mimic the inhomogeneous broadening of the actual ensemble) is valid. Most importantly, this simplified model allows us to directly reveal the plasmonic nature of such lobes. Actually, our model is similar, apart from the extra broadening caused by inhomogeneities, to the single nanoparticle model employed for monodispersed ensembles of gold nanospheres [49] and 
single (isolated) gold nanorods [50,51]. In a single plasmonic nanostructure, the Fermi smearing of the electrons energy distribution induces a red-shift of the plasmonic resonance in the extinction cross-section, caused by an increase of the real part of the gold dielectric function in the red-infrared spectral region, which is known to be proportional to the temperature $T_{E}(t)$ of the thermalized electrons [49]. This shift translates into a $\Delta T / T$ spectrum with a characteristic derivative behavior: the transmittance decreases at longer wavelengths (where the extinction is increased as a result of the redshift of the plasmon) and increases at shorter wavelengths (where the extinction is decreased). This is the dominating effect on the first few picosecond time scale, although deviations from a purely derivative behavior can be observed because of the contribution to the optical response arising from nonthermalized carriers (see below for further details) and (on a longer time scale) from the heating of the lattice [50,51]. In our metasurface the plasmonic peak (for the orthogonal polarization) is located at $820 \mathrm{~nm}$ (cf. Fig. 2) and is very broad, so the pump-induced red-shift of the plasmon causes the appearance of the positive lobe peaked at $700 \mathrm{~nm}$ and of the negative lobe peaked at around $1000 \mathrm{~nm}$ in the differential transmission map of Fig. 2(b). The asymmetry of the two lobes arises mostly because of the high dispersion of the metal dielectric function (and it is thus more pronounced for broader plasmonic resonances, as in our case). Of course the agreement between the experimental map [Fig. 3(b)] and the theoretical one [Fig. 3(d)] is not fully quantitative for the orthogonal polarization, and in particular we miss to reproduce some spectral features in the 400-600-nm wavelength range and the exact decay time of the dynamics. However, the mismatch is modest, especially considering the simplification introduced with Eq. (3) and the effective inhomogeneous broadening of the plasmonic resonance which was mimicked by simply adding a large offset $\Delta \epsilon_{I}=15$ to the imaginary part of gold permittivity (cf. Sec. II). Also, it is interesting to compare our dynamical model, based on the ETTM, with the simpler phenomenological TTM, which can be viewed as the limiting case of the ETTM with instantaneous electronelectron thermalization, i.e., $H(t)=\delta(t)$ in Eq. (4), $\delta(t)$ being the Dirac delta function. The substantial lack of the TTM is within the subpicosecond time scale where the interplay between nonthermalized and thermalized carriers can give rise, at certain wavelengths, to complex dynamical features in the $\Delta T / T$ signal, that can not be reproduced by the TTM. For example, ultrafast oscillations (with sign changes) can be observed in the visible [Fig. 4(a)] and ultrafast $\Delta T / T$ decay is seen in the infrared wing of the transient plasmonic response [Fig. 4(b)], whereas close to the plasmonic peak, the mismatch between the two models is mostly quantitative [Fig. 4(c)]. The discrepancy between the two theoretical predictions is ascribable to the effect of nonthermalized carriers, which is totally disregarded in the TTM model.

\section{ALL-OPTICAL MODULATION PERFORMANCE}

We also investigated the performance of the metasurface as an ultrafast transmission modulator in the red/infrared. Figure 5 shows the dynamics of the $\Delta T / T$ signal recorded at $700-\mathrm{nm}$ wavelength [i.e., within the broad positive lobe
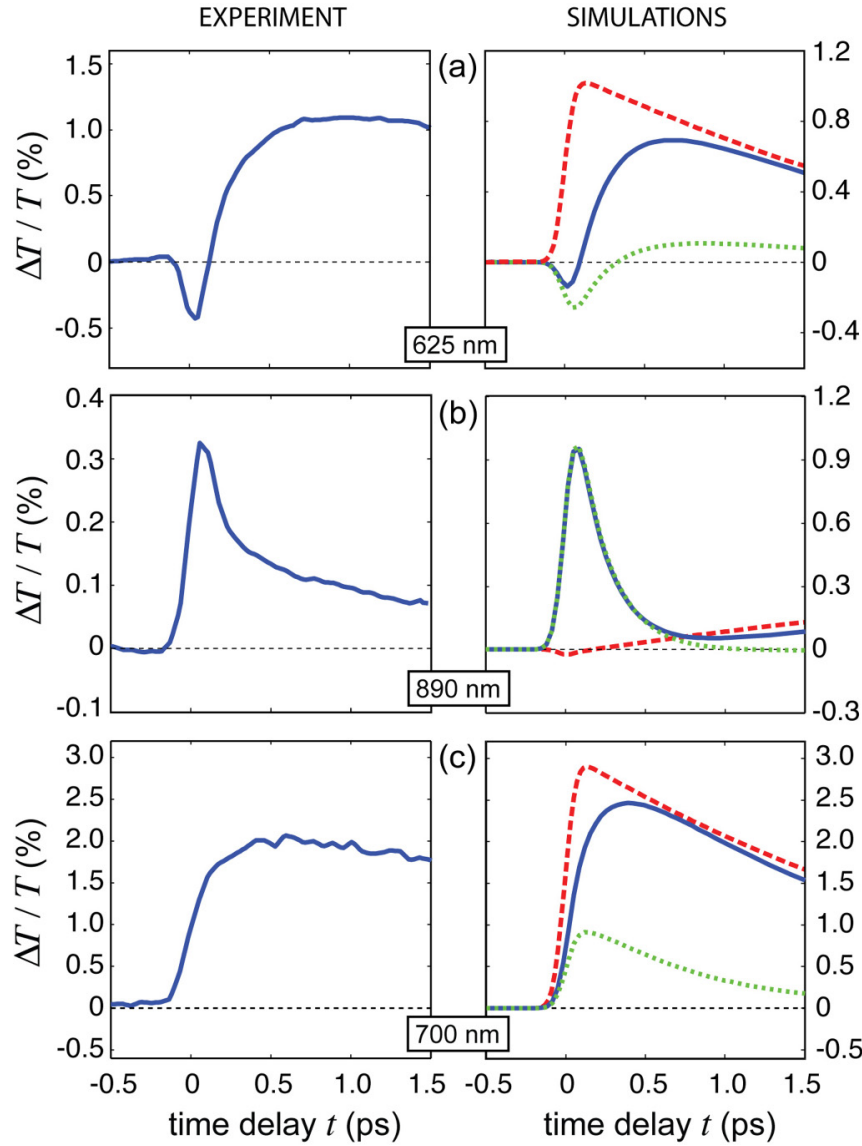

FIG. 4. (Color online) $\Delta T / T$ dynamics for the orthogonal polarization under pump incident fluence $F=0.6 \mathrm{~mJ} / \mathrm{cm}^{2}$ at different probe wavelengths: (a) $\lambda=625$, (b) 890 , and (c) $700 \mathrm{~nm}$. Left panels show the experimental results [after Fig. 3(b)]. Right panels show the theoretical $\Delta T / T$ according to the ETTM (blue solid curve) and according to the simpler TTM (red dashed curve). The green dotted curve represents the contribution to the $\Delta T / T$ arising from nonthermalized carriers within the ETTM.

of Fig. 3(b)] under different pump fluences for (a) parallel and (b) orthogonal polarizations. Note that the $\Delta T / T$ peak is strongly enhanced (in absolute value) under the orthogonal polarization as compared with the parallel polarization [inset in Fig. 5(a)], thanks to the onset of the plasmonic resonance of the metasurface peaked at around $820 \mathrm{~nm}$.

At $3.7-\mathrm{mJ} / \mathrm{cm}^{2}$ pump fluence, the maximum $\Delta T / T$ value for the orthogonal polarization turned out to be close to $10 \%$, which is a promising result towards the development of all-optical modulators based on self-organized plasmonic metasurfaces. However, a further increase of the pump fluence seems to have a marginal effect; at $9-\mathrm{mJ} / \mathrm{cm}^{2}$ fluence, the maximum $\Delta T / T$ was $10.5 \%$. Note that this trend is intrinsically related to the plasmonic response of the metasurface, because it is not observed for the parallel polarization [Fig. 5(a)]. However, when plotting the spectral cross-sections of the maps at the time delay of the signal peak $(\sim 0.5 \mathrm{ps})$ for different pump fluences [Figs. 5(c) and 5(d)] a deformation of the transient plasmonic spectrum is revealed. The peak of the signal at $9 \mathrm{~mJ} / \mathrm{cm}^{2}$ pump fluence is blue-shifted to $650 \mathrm{~nm}$ and is as high as $15 \%$. Such a deformation of the spectra is not visible 

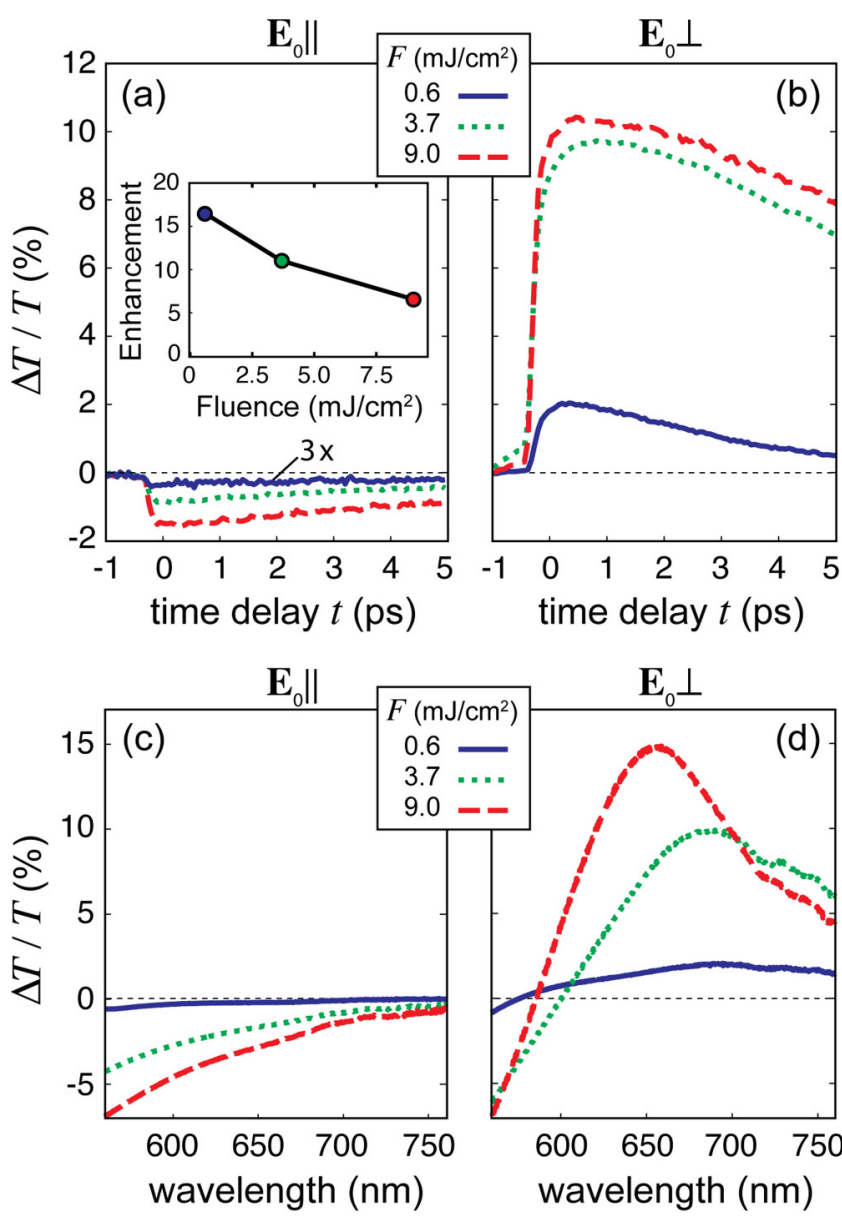

FIG. 5. (Color online) Temporal cross-sections of the $\Delta T / T$ experimental map at $\lambda=700 \mathrm{~nm}$ probe wavelength under different pump fluences for (a) parallel polarization and (b) orthogonal polarization. The inset in (a) shows the enhancement (in absolute value) of the $\Delta T / T$ value achieved for orthogonal polarization with respect to parallel polarization, computed as the ratio between peak values in (b) and (a) respectively, at a given pump fluence. Spectral cross-sections of the $\Delta T / T$ experimental map at $\sim 0.5$-ps time delay under different pump fluences for (c) parallel polarization and (d) orthogonal polarization.

for the parallel polarization [Fig. 5(c)] and is again plasmonic in nature. A detailed explanation of this effect is beyond the scope of the present paper, and is an indication of the onset of a non perturbative regime of the pump-induced excitation, which cannot be captured by the perturbative models so far reported in the literature. Finally, note that under $9-\mathrm{mJ} / \mathrm{cm}^{2}$ pump fluence the FWHM of the $\Delta T / T$ positive lobe exceeds $100 \mathrm{~nm}$, indicating the potential of the metasurface to operate as a broad-band all-optical modulator. Considering the broader operation band, the energy cost of $9 \mathrm{~mJ} / \mathrm{cm}^{2}$ per pump pulse required to achieve $15 \%$ modulation is in line with the state of the art in the field of all-optical modulation from plasmonic metasurfaces (see, e.g., Ref. [16]).

\section{CONCLUSION}

We experimentally demonstrated that a self-organized metasurface produced by defocused $\mathrm{Ar}^{+}$ion beam sputtering is capable of providing high polarization-sensitive transmission under excitation with fs laser pulses. The metasurface can be described as an ensemble of parallel gold NWs with very high aspect ratio and dispersed in width and height. This allowed us to introduce a simplified polarization-dependent two-dimensional model to simulate the linear and nonlinear optical response of the structure. A clear-cut identification of the plasmonic mechanisms governing the electromagnetic properties of the metasurface is achieved. We found that under excitation with electric field parallel to the major axis of the NWs the metasurface effectively behaves as a homogenous thin gold film, showing no plasmonic response. On the contrary, for the orthogonal polarization the metasurface exhibits an intense and broadband plasmonic resonance in the near infrared, thanks to the excitation of the transverse plasmonic mode in the highly dispersed population of NWs. Since we found experimentally a relative differential transmission as high as $15 \%$ in the red/infrared under moderate pump fluence (i.e., below $10 \mathrm{~mJ} / \mathrm{cm}^{2}$ ), we envision that $\mathrm{Ar}^{+}$ ion beam sputtering can represent a valuable, simple and low-cost method for the realization of self-organized large area plasmonic metasurfaces with potential applications to all-optical modulation and switching.

\section{ACKNOWLEDGMENTS}

Financial support from the Fondazione Cariplo is acknowlegded by G.D.V. and S.L. through the project New Frontiers in Plasmonic Nanosensing (Grant No. 2011-0338). G.D.V., M.F. and P.B. acknowledge the COST Action MP1302NanoSpectroscopy. G.C. acknowledges support by the EC under Graphene Flagship (Contract No. CNECT-ICT-604391). F.B.d.M. acknowledges support by MAE in the framework of the Italia-Polonia bilateral program, and by Compagnia di San Paolo in the framework of project ID ROL 1228. Financial support by Ministero dell'Università e della Ricerca Scientifica through the PRIN project No. 2008J858Y7 is gratefully acknowledged.

\section{APPENDIX A: THE NEED FOR A LARGE SET OF SIZES}

The extinction of a nano-object scales with its volume (under quasistatic approximation the absorption is linearly proportional to the volume and the scattering is proportional to the volume squared). To elucidate the need for taking into account the large set of sizes comprised in the distribution $\rho$ of Fig. 1(c), one should inspect the weighted size distribution $\rho_{V}$ obtained by multiplying each pixel in the map of Fig. 1(c) with the volume $w h$ of the corresponding nanowire.

This is shown in Fig. 6. Note that, contrary to $\rho$, in the map of $\rho_{V}$ there is no evidence of a highly representative wire, meaning that it is not possible to exclude $a$ priori the thick and wide NWs in the simulation of the linear optical response of the metasurface [Eq. (2)], although these wires are very rare in terms of $\rho$ [cf. Fig. 1(c)]. These NWs, can contribute to the optical response, depending on their resonance wavelength. In general, having plasmonic resonances out of the spectral region of Fig. 2(b), they contribute to the broadening of the dip at $820 \mathrm{~nm}$ and to the onset of an almost flat background. 


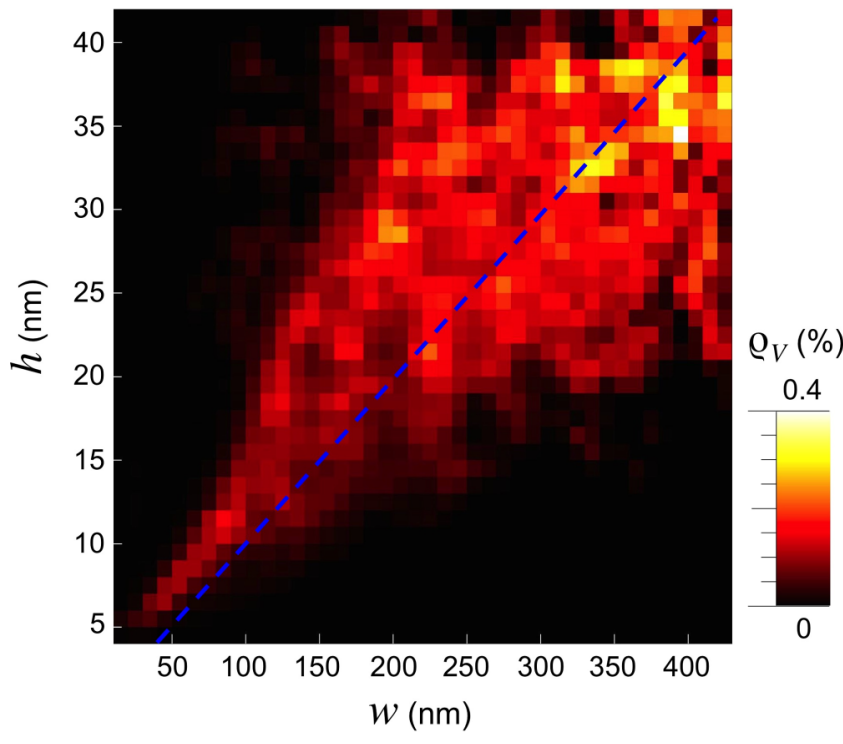

FIG. 6. (Color online) Volume weighted size distribution $\rho_{V}(w, h)=k \rho(w, h) w h, k$ being a normalization constant.

\section{APPENDIX B: COMPARISON WITH AN ARRAY MODEL}

In this appendix, we discuss a different heuristic model for the optical response of the metasurface under orthogonal polarization. Instead of an individual inhomogeneously broadened NW [Eq. (3)], we consider a linear array of NWs. Two NW sizes are investigated: the one used in the model of the transient optical response of Sec. III B, i.e., $w=110 \mathrm{~nm}$ and $h=8.5 \mathrm{~nm}$, and the size corresponding to the most representative wire in the distribution of Fig. 1(c), i.e., $w=50 \mathrm{~nm}$ and $h=6 \mathrm{~nm}$. The results are reported in Figs. 7(a) and 7(b), respectively. Different values of the gap distance $g$ between adjacent NWs are considered. For each value of $g$ the array period is set to $\Lambda=w+g$. Note that for $w=110 \mathrm{~nm}, h=8.5 \mathrm{~nm}$ and $g=g_{\text {ave }}=50 \mathrm{~nm}$ [black dotted curve in Fig. 7(a)], the peak wavelength and width of the plasmonic spectrum approximately match the results of the full model of Eq. (2) (red solid curve). The array model thus reproduces the broadening of the plasmonic response of the metasurface by exploiting the near-field coupling between identical NWs (which is well-known to induce broadening of the plasmonic resonance [52]) without recurring to the heuristic estimation of the inhomogeneous broadening used in the single NW model of Eq. (3). However, the minimum value of the transmittance at the plasmonic peak is as low as $1 \%$, meaning that an heuristic correction (i.e., a rescaling of the power extinction by a fitting

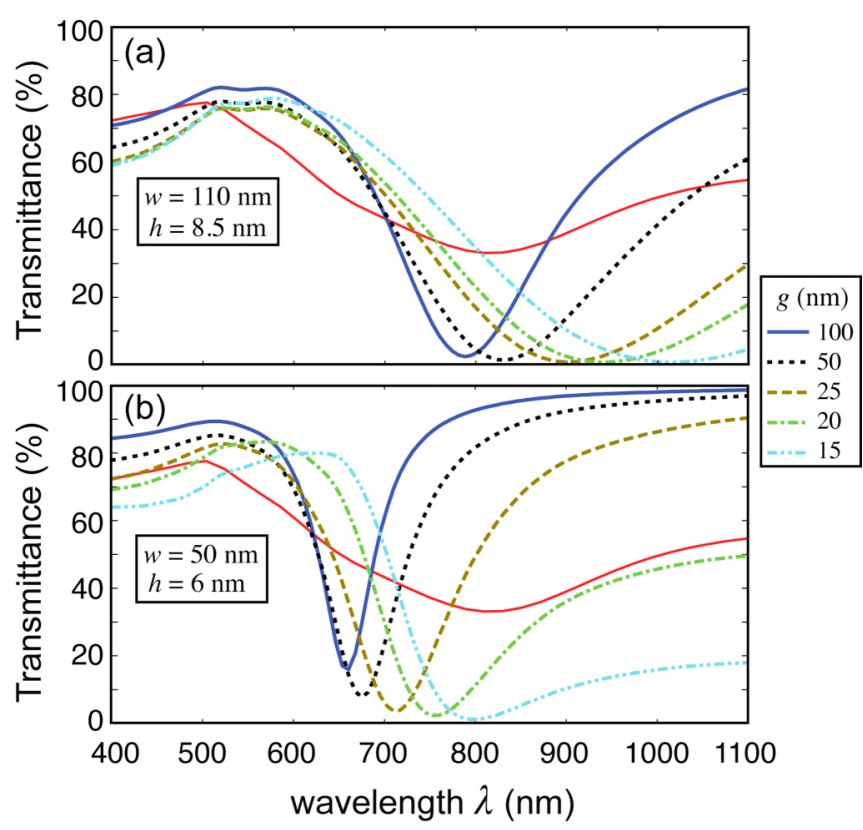

FIG. 7. (Color online) FEM numerical simulation of the transmittance of homogeneous arrays of single-sized gold NWs embedded into an effective medium with $\epsilon_{\text {eff }}=1.56$. (a) $w=100 \mathrm{~nm}, h=8.5$ $\mathrm{nm}$; (b) $w=50 \mathrm{~nm}, h=6 \mathrm{~nm}$. The transmittance is computed as $T=P_{\text {out }} /\left(I_{i n} \Lambda\right), I_{\text {in }}$ being the incident light intensity, $\Lambda$ the array period, and $P_{\text {out }}$ the total power that flows across a horizontal line of length $\Lambda$ placed $1 \mu \mathrm{m}$ below the array. Periodic boundary conditions are imposed on the left and right sides of the array cell, whereas perfectly-matched layers are employed to treat the open boundaries at the top and bottom sides. Solid red curve is the full model computation according to Eq. (2).

parameter $\xi$ ) is nevertheless required to make the array model realistic, similarly to the case of Eq. (3). One can finally notice that, when $w=50 \mathrm{~nm}$ and $h=6 \mathrm{~nm}$ are considered, the array model requires a much smaller gap between the wires, i.e., $g=15 \mathrm{~nm}$ or less [cyan double dot-dashed curve in Fig. 7(b)] in order to match the correct peak wavelength and width of the plasmonic spectrum. Moreover, the shape of the spectrum exhibits major deviations (the left wing is steeper whereas the right wing is smoother) to the correct behavior (red solid curve). This counter-intuitive result supports the conclusion that, for modeling the optical response of the metasurface, one should not select the most representative wire in the size distribution. The total optical response is instead the result of the interplay between different factors: abundance in the size distribution, NW volume, and spectral position of the extinction peak of each plasmonic NW.
[1] H. Raether, Surface Plasmons (Springer-Verlag, Berlin, 1986)

[2] J. A. Schuller, E. S. Barnard, W. Cai, Y. C. Jun, J. S. White, M. 1. Brongersma, Nat. Mater. 9, 193 (2010).

[3] S. Lal, S. Link, and N. J. Halas, Nat. Photon. 1, 641 (2007).

[4] P. Biagioni, J.-S. Huang, and B. Hecht, Prog. Phys. 75, 024402 (2012).
[5] N. Yu and F. Capasso, Nat. Mater. 13, 139 (2014).

[6] A. Pors, M. G. Nielsen, G. Della Valle, M. Willatzen, O. Albrektsen, and S. I. Bozhevolnyi, Opt. Lett. 36, 1626 (2011).

[7] Y. Zhao and A. Alú, Phys. Rev. B 84, 205428 (2011).

[8] E. Skovsen, T. Sondergaard, C. Lemke, T. Holmgaard, T. Leißner, R. L. Eriksen, J. Beermann, M. Bauer, K. Pedersen, and S. I. Bozhevolnyi, Appl. Phys. Lett. 103, 211102 (2013). 
[9] X. Ni, N. K. Emani, A. V. Kildishev, A. Boltasseva, and V. M. Shalaev, Science 335, 427 (2012).

[10] A. Pors, M. G. Nielsen, and S. I. Bozhevolnyi, Nano Lett. 15, 791 (2015).

[11] M. Kauranen and A. V. Zayats, Nat. Photon. 6, 737 (2012).

[12] D. D. Smith, Y. Yoon, R. W. Boyd, J. K. Campbell, L. A. Baker, R. M. Crooks, and M. George, J. Appl. Phys. 86, 6200 (1999).

[13] N. Rotenberg, A. D. Bristow, M. Pfeiffer, M. Betz, and H. M. van Driel, Phys. Rev. B 75, 155426 (2007).

[14] C.-K. Sun, F. Vallée, L. H. Acioli, E. P. Ippen, and J. G. Fujimoto, Phys. Rev. B 50, 15337 (1994).

[15] X. Zhang, B. Sun, J. M. Hodgkiss, and R. H. Friend, Adv. Mater. 20, 4455 (2008).

[16] G. A. Wurtz, R. Pollard, W. Hendren, G. P. Wiederrecht, D. J. Gosztola, V. A. Podolskiy, and A. V. Zayats, Nat. Nanotechnol. 6, 107 (2011).

[17] J. A. Fan, C. Wu, K. Bao, J. Bao, R. Bardhan, N. J. Halas, V. N. Manoharan, P. Nordlander, G. Shvets, and F. Capasso, Science 328, 1135 (2010).

[18] R. Verre, K. Fleischer, O. Ualibek, and I. V. Shvets, Appl. Phys. Lett. 100, 031102 (2012).

[19] Y. Liu and X. Zhang, Chem. Soc. Rev. 40, 2494 (2011).

[20] A. Toma, D. Chiappe, B. Sětina Batič, M. Godec, M. Jenko and F. Buatier de Mongeot, Phys. Rev. B 78, 153406 (2008).

[21] A. Toma, B. Sětina Batič, D. Chiappe, C. Boragno, U. Valbusa, M. Godec, M. Jenko and F. Buatier de Mongeot, J. Appl. Phys. 104, 104313 (2008).

[22] A. Toma, D. Chiappe, C. Boragno, and F. Buatier de Mongeot, Phys. Rev. B 81, 165436 (2010).

[23] D. Chiappe, A. Toma and F. Buatier de Mongeot, Small 9, 913 (2013).

[24] L. Novotny and C. Hafner, Phys. Rev. E 50, 4094 (1994).

[25] T. Sondergaard and S. I. Bozhevolnyi, Phys. Rev. B 75, 073402 (2007).

[26] COMSOL MULTIPHYSICS 3.5a.

[27] T. Sondergaard, J. Jung, S. I. Bozhevolnyi and G. Della Valle, New J. Phys. 10, 105008 (2008).

[28] The FEM computation of all the extinction cross-section spectra required to implement Eq. (2) takes about two days on a modern multicore workstation.

[29] P. Yeh, Optical Waves in Layered Media (Wiley, New York, 1988).

[30] P. B. Johnson and R. W. Christy, Phys. Rev. B 6, 4370 (1972).

[31] U. Kreibig and U. Genzel, Surf. Sci. 156, 678 (1985).

[32] P. Yu. Kuryoz, L. V. Poperenko, and V. G. Kravets, Phys. Status Solidi A 210, 2445 (2013).

[33] K.-P. Chen, V. P. Drachev, J. D. Borneman, A. V. Kildishev, and V. M. Shalaev, Nano Lett. 10, 916 (2010).
[34] C. Manzoni, D. Polli, and G. Cerullo, Rev. Sci. Instrum. 77, 023103 (2006).

[35] D. Polli, L. Luer, and G. Cerullo, Rev. Sci. Instrum. 78, 103108 (2007).

[36] G. Della Valle, M. Conforti, S. Longhi, G. Cerullo, and D. Brida, Phys. Rev. B 86, 155139 (2012).

[37] S. Dal Conte, M. Conforti, D. Petti, E. Albisetti, S. Longhi, R. Bertacco, C. De Angelis, G. Cerullo, and G. Della Valle, Phys. Rev. B 89, 125122 (2014).

[38] N. Del Fatti, R. Bouffanais, F. Vallée, and C. Flytzanis, Phys. Rev. Lett. 81, 922 (1998).

[39] S. I. Anisimov, B. L. Kapeliovich, and T. L. Perel'man, Sov. Phys. JETP 39, 375 (1974).

[40] G. Della Valle, F. Scotognella, A. R. S. Kandada, M. ZavelaniRossi, H. Li, M. Conforti, S. Longhi, L. Manna, G. Lanzani, and F. Tassone, J. Phys. Chem. Lett. 4, 3337 (2013).

[41] R. H. M. Groeneveld, R. Sprik, and A. Lagendijk, Phys. Rev. B 51, 11433 (1995).

[42] W. S. Fann, R. Storz, H. W. K. Tom, and J. Bokor, Phys. Rev. B 46, 13592 (1992).

[43] E. Carpene, Phys. Rev. B 74, 024301 (2006).

[44] F. Scotognella, G. Della Valle, A. R. S. Kandada, D. Dorfs, M. Zavelani-Rossi, M. Conforti, K. Miszta, A. Comin, K. Korobchevskaya, G. Lanzani, L. Manna, and F. Tassone, Nano Lett. 11, 4711 (2011).

[45] Contrary to our previous paper [37], here we disregarded the contribution to $\Delta \epsilon_{\mathrm{Au}}(\lambda, t)$ arising from interband transition around the $X$ point, since it is known to be much weaker as compared with the contribution from interband transitions around the $L$ point.

[46] F. Masia, W. Langbein, and P. Borri, Phys. Rev. B 85, 235403 (2012).

[47] A. Alabastri, S. Tuccio, A. Giugni, A. Toma, C. Liberale, G. Das, F. De Angelis, E. Di Fabrizio, and R. Proietti Zaccaria, Mater. 6, 4879 (2013).

[48] A. Marini, M. Conforti, G. Della Valle, H. Lee, T. X. Tran, W. Chang, M. A. Schmidt, S. Longhi, P. St. J. Russell and F. Biancalana, New. J. Phys. 15, 013033 (2013).

[49] N. Del Fatti, F. Vallée, C. Flytzanis, Y. Hamanaka, and A. Nakamura, Chem. Phys. 251, 215 (2000).

[50] H. Baida, D. Mongin, D. Christofilos, G. Bachelier, A. Crut, P. Maioli, N. Del Fatti, and F. Vallée, Phys. Rev. Lett. 107, 057402 (2011).

[51] M. Zavelani-Rossi, D. Polli, S. Kochtcheev, A.-L. Baudrion, J. Beál, V. Kumar, E. Molotokaite, M. Marangoni, S. Longhi, G. Cerullo, P.-M. Adam, and G. Della Valle, ACS Photonics 2, 521 (2015).

[52] L.-L. Zhao, K. L. Kelly, and G. C. Schatz, J. Phys. Chem. B 107, 7343 (2003). 